\title{
GENERALIZATION OF GOLDEN-THOMPSON TYPE INEQUALITIES FOR NORMAL MATRICES
}

\author{
XUHUA LiU
}

Abstract. We survey some well-known matrix exponential formulae, with emphasis on logmajorization results, by using the compound matrix method.

Mathematics subject classification (2010): 15A45, 15B48.

Keywords and phrases: Golden-Thompson inequality, Araki-Lieb-Thirring inequality, Bernstein inequality, Cordes inequality, normal matrices, trace, log-majorization.

\section{REFERENCES}

[1] T. ANDO, F. HiAI, Log majorization and complementary Golden-Thompson type inequalities, Linear Algebra Appl. 197/198 (1994), 113-131.

[2] H. Araki, On an inequality of Lieb and Thirring, Lett. Math. Phys. 19 (1990), 167-170.

[3] N. Bebiano, J. DA PRovidÊncia, R. Lemos, Matrix inequalities in statistical mechanics, Linear Algebra Appl. 376 (2004), 265-273.

[4] D. S. BERnSTEIn, Inequalities for the trace of matrix exponentials, SIAM J. Matrix Anal. Appl. 9 (1988), 156-158.

[5] R. Bhatia, Matrix Analysis, Springer-Verlag, New York, 1997.

[6] J.-C. BouRIn, E.-Y. LEE, Matrix inequalities from a two variables functional, Int. J. Math (to appear) http://dx.doi.org/10.1142/S0129167X16500713

[7] J. E. CoHEN, Spectral inequalities for matrix exponentials, Linear Algebra Appl. 111 (1988), 25-28.

[8] J. E. Cohen, S. Friedland, T. Kato, F. Kelly, Eigenvalue inequalities for products of matrix exponentials, Linear Algebra Appl. 45 (1982), 55-95.

[9] H. O. Condes, Spectral Theory of Linear Differential Operators and Comparison Algebras, Cambridge University Press, (1987).

[10] K. FAN, On a theorem of Weyl concerning eigenvalues of linear transformations, I, Proc. Nat. Acad. Sci. USA 35 (1949), 652-655.

[11] K. FAN, Maximum properties and inequalities for the eigenvalues of completely continuous operators, Proc. Nat. Acad. Sci. USA 37 (1951), 760-766.

[12] P. J. Forrester, C. J. ThOMPSON, The Golden-Thompson inequality: historical aspects and random matrix applications, J. Math. Phys. 55 (2014), 023503.

[13] S. Golden, Lower bounds for Helmholtz function, Phys. Rev. 137 (1965), B1127-B1128.

[14] F. HIAI, Trace norm convergence of exponential product formula, Lett. Math. Phys. 33 (1995), 147158.

[15] F. HiAI, Log-majorizations and norm inequalities for exponential operators, Linear Operators (Warsaw, 1994), 119-181, Banach Center Publ. 38, Polish Acad. Sci., Warsaw, 1997.

[16] F. HiAI, D. PETz, The Golden-Thompson trace inequality is complemented, Linear Algebra Appl. 181 (1993), 153-185.

[17] A. Horn, On the eigenvalues of a matrix with prescribed singular values, Proc. Amer. Math. Soc., 5 (1954), 4-7.

[18] B. Kostant, On convexity, the Weyl group and the Iwasawa decomposition, Ann. Sci. École Norm. Sup. (4) 6 (1973), 413-455.

[19] L. LenARD, Generalization of the Golden-Thompson inequality $\operatorname{tr}\left(e^{A} e^{B}\right) \geqslant \operatorname{tr} e^{A+B}$, Indiana Univ. Math. J. 21 (1971), 457-467. 
[20] E. LIEB, W. ThIRring, Inequalities for the moments of the eigenvalues of the Schrödinger hamiltonian and their relation to sobolev inequalities, Studies in Mathematical Physics, 269-303, Princeton University Press, 1976.

[21] M. MarCus, Finite Dimensional Multilinear Algebra I, Marcel Dekker, 1973.

[22] M. MARCus, Finite Dimensional Multilinear Algebra II, Marcel Dekker, 1975.

[23] R. MERris, Multilinear Algebra, Gordon and Breach Science Publishers, 1997.

[24] D. PETZ, A survey of certain trace inequalities, Functional Analysis and Operator Theory (Warsaw, 1992), 287-298, Banach Center Publ. 30, Polish Acad. Sci., Warsaw, 1994.

[25] W. So, Equality cases in matrix exponential inequalities, SIAM J. Math. Anal. 13 (1992), 1154-1158.

[26] K. SyMANZIK, Proof of refinements of an inequality of Feynmann, J. Math. Phys. 6 (1965), 11551156.

[27] C. J. Thompson, Inequality with applications in statistical mechanics, J. Math. Phys. 6 (1965), $1812-1813$.

[28] C. J. Thompson, Inequalities and partial orders on matrix spaces, Indiana Univ. Math. J. 21 (1971), 469-480.

[29] J. von Neumann, Some matrix-inequalities and metrization of matric-space, Tomsk. Univ. Rev., 1 (1937), 286-300. 